\title{
Efecto de la labranza en la estabilidad estructural y resistencia a la penetración en un Inceptisol sembrado con arracacha (Arracacia xanthorrhiza Bancroft) en Boyacá
}

\section{Effect of tillage on the structural stability and resistance to penetration of a Inceptisol planted with arracacha (Arracacia xanthorrhiza Bancroft) in Boyaca}
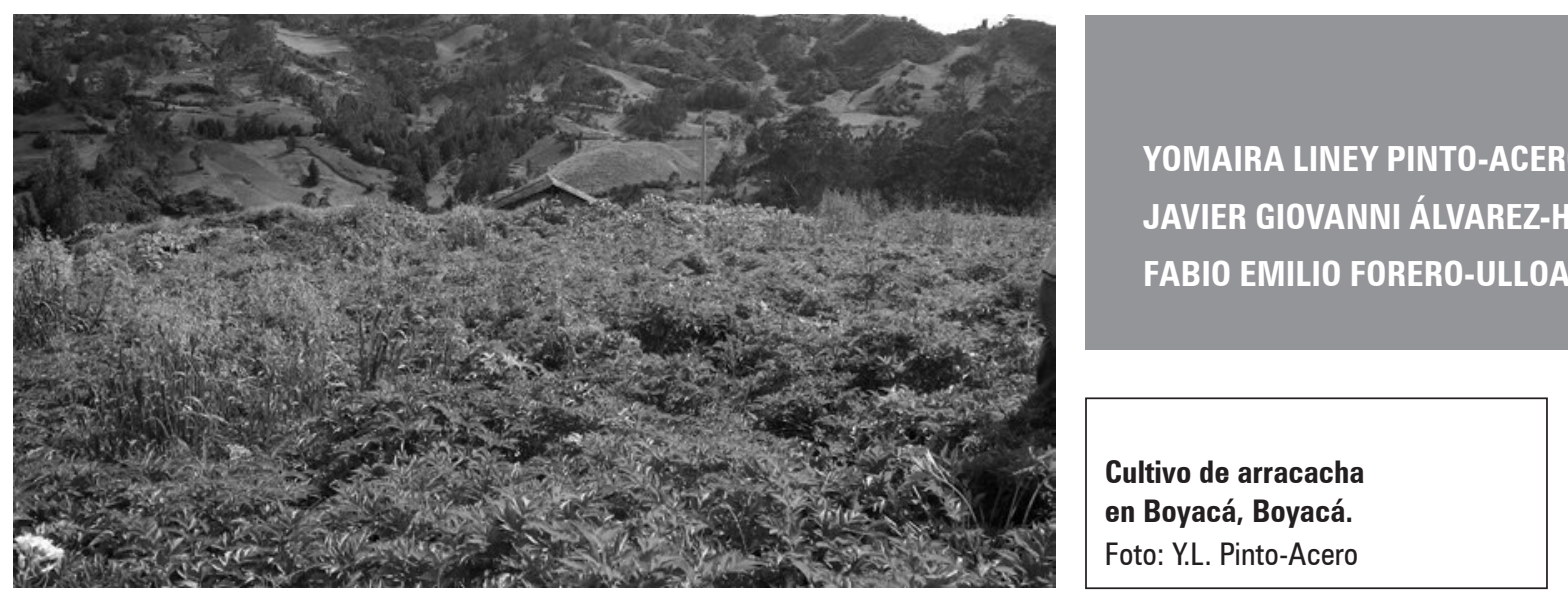

\section{RESUMEN}

En el municipio de Boyacá (departamento de Boyacá, Colombia), la producción de arracacha constituye una fuente de ingresos importante para los agricultores. Debido a la escasa información sobre el paquete tecnológico para este cultivo, se estudió el efecto de diferentes sistemas de labranza (labranza mínima, labranza convencional y labranza vertical) sobre algunas de las principales propiedades físicas de un suelo Typic Dystrudepts, con pendiente del $3 \%$, tipo de paisaje montañoso y tipo de relieve lomerío; durante cuatro épocas de muestreo (antes de la preparación (E1), después de la preparación (E2), 100 días después de la siembra (E3) y 180 días después de la siembra (E4)). El diseño fue en bloques completamente aleatorizados, donde cada bloque correspondió a un tipo de labranza, y los tratamientos fueron dos materiales de arracacha (Yema de huevo y Paliverde). Los valores más bajos de resistencia a la penetración se presentaron en la labranza vertical a los 12,$5 ; 17,5$ y $20 \mathrm{~cm}$, con valores de 262,8; 810,8 y 1337,5 kPa respectivamente, del mismo modo, este tipo de labranza presentó una mayor estabilidad estructural y un gran porcentaje de distribución de agregados de tamaño entre 1 y $3 \mathrm{~mm}$, con un valor promedio de $31,4 \%$, frente a la labranza convencional y la labranza mínima, con valores de $22 \%$ y $25,2 \%$ respectivamente, lo cual favoreció el desarrollo radicular de las plantas de arracacha. La mayor diferencia entre los tipos de labranza para la distribución por tamaño de agregados se presentó a los 100 días después de la siembra. Los materiales de arracacha evaluados no afectaron de forma significativa ninguno de los parámetros del suelo.

Facultad de Ciencias Agropecuarias, Grupo de Investigaciones Agrícolas, Universidad Pedagógica y Tecnológica de Colombia, Tunja (Colombia).

2 Facultad de Ciencias Agropecuarias, Universidad Pedagógica y Tecnológica de Colombia, Tunja (Colombia).

Autor para correspondencia. jgalvarezh@gmail.com 


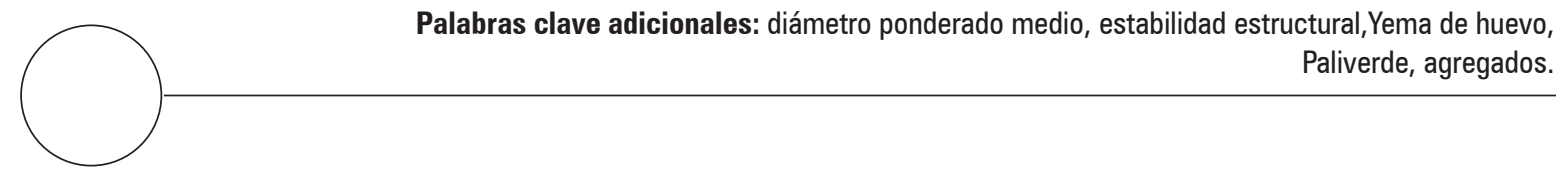

\section{ABSTRACT}

In the municipality of Boyaca (Boyaca, Colombia), arracacha production constitutes an important source of income for farmers. Because of the limited information on the technology used for this crop, the effect of different tillage systems (minimum tillage, conventional tillage and vertical tillage) on some of the main physical properties of a Typic Dystrudept was studied, with a slope of $3 \%$, mountainous type landscape and hilly relief, using four sampling times (before preparation (E1), after preparation (E2), 100 days after planting (E3) and 180 days after planting (E4)). The design employed a randomized block, wherein each block corresponded to a type of farming and the treatments used two arracacha types (Yema de huevo and Paliverde). The lower values of penetration resistance were seen with the vertical tillage at 12.5, 17.5 and 20 $\mathrm{cm}$ with values of $262.8,810.8$ and $1337.5 \mathrm{kPa}$, respectively. Furthermore, the vertical tillage had a greater increased structural stability and larger percentage of aggregate distribution for sizes between 1 and $3 \mathrm{~mm}$, with an average value of $31.4 \%$, than the conventional tillage and minimum tillage, which had values of $22 \%$ and $25.2 \%$, respectively, which favored root development in the arracacha plants. The biggest difference between the types of tillage in terms of aggregate size distribution was seen at 100 days after sowing. The evaluated arracacha materials did not significantly affect any of the evaluated soil parameters.

Additional keywords: weighted mean diameter, structural stability, yema de huevo, Paliverde, aggregates.

Fecha de recepción: 09-02-2016

Aprobado para publicación: 14-05-2016
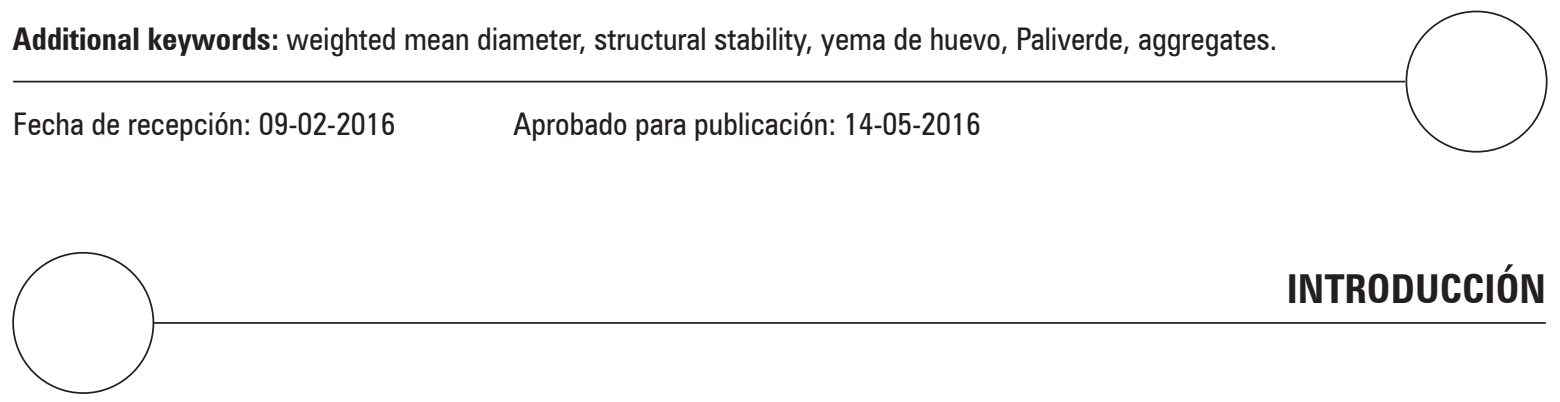

De las 714 ha sembradas con arracacha en el departamento de Boyacá (Colombia), el 28\% del área cultivada se encuentra en el municipio de Boyacá. Su rendimiento promedio en $\mathrm{kg} \mathrm{ha}^{-1}$ es superior en un $25 \%$ al establecido a nivel departamental (9.018 $\left.\mathrm{kg} \mathrm{ha}^{-1}\right)$, frente a los $12.000 \mathrm{~kg} \mathrm{ha}^{-1}$ obtenidos a nivel local (EOT, 2007). Sin embargo, esta especie que ha sido de importancia económica en regiones con tipo de tenencia de tierra minifundista no ha sido objeto de la aplicación de tecnologías de mejoramiento tanto en el manejo de las labores de cultivo como en la cosecha o en su industrialización (Alvarado y Ochoa, 2010).

Una de las variables que más afecta el crecimiento óptimo de la arracacha es la resistencia del suelo a la penetración, debido a que este atributo influye en el desarrollo radicular (Cortés et al., 2013), así mismo, se conoce que en algunos suelos a mayor profundidad en el perfil, este tiende a ser más compacto (Keller et al., 2013). También es sabido que la resistencia a la penetración es una de las propiedades que más se ve afectada por la mecanización (Ramírez-López et al., 2008), debido a que al labrar un suelo este cambia propiedades como la densidad aparente, porosidad y aireación. Por otro lado, un suelo excesivamente cultivado puede llegar a presentar problemas de compactación, lo cual conduce a cambios en el desarrollo de las raíces, y puede llegar a limitar la absorción de nutrientes (Casanova y Lobo, 2007). Otro factor importante es la 
estabilidad estructural debido a que la susceptibilidad del suelo está estrechamente relacionada con esta (Barthes y Roose 2002). La mecanización de un suelo tiende a que el diámetro ponderado medio sea más bajo que sin mecanización (Safar et al., 2011).

Con base en lo anterior, el objetivo de este trabajo fue determinar la relación entre la resistencia a la penetración y la estabilidad estructural del suelo, observando diferentes tipos de labranza para la siembra de arracacha, de los materiales Yema de huevo y Paliverde, durante los primeros días de crecimiento del cultivo.

\section{MATERIALES Y MÉTODOS}

La fase de campo del estudio se desarrolló en la vereda Huerta Grande en el municipio de Boyacá (Boyacá), situado a 5¹1'40"N y 7308'44"W, a una altitud de $2.400 \mathrm{msnm}$ con una precipitación de $1.477 \mathrm{~mm}$ acumulados en el periodo comprendido entre los meses de mayo a diciembre de 2014. Los datos de temperatura registrados indican que la temperatura media fue de $11^{\circ} \mathrm{C}$ y la humedad relativa promedio fue de $80 \%$ (Ideam, 2014). El tipo de suelo identificado fue Typic Dystrudepts (tabla 1), con una pendiente del $3 \%$, tipo de paisaje montañoso y tipo de relieve lomerío, altamente representativo para la zona productora de arracacha. La fase de laboratorio se realizó en los laboratorios de suelos de la Facultad de Ciencias Agropecuarias de la Universidad Pedagógica y Tecnológica de Colombia, Tunja.

Se utilizaron 450 colinos de material Paliverde y 450 colinos de material Yema de huevo, debido a su alta comercialización y producción en la zona de estudio. La preparación del suelo se realizó con un tractor Kubota M9540, con arado de discos (LC), cincel vibratorio (LV) y azadón (LM).

El diseño experimental fue en bloques completamente aleatorizados, en donde cada bloque correspondió a un tipo de labranza. Se establecieron dos tratamientos (yema de huevo y paliverde) con tres repeticiones para un total de seis unidades experimentales (UE) por bloque. Cada bloque tenía una medida de $6 \mathrm{~m}$ x $24 \mathrm{~m}$, y cada UE tenía un tamaño de $24 \mathrm{~m}^{2}$.

La resistencia a la penetración se determinó con un penetrómetro cónico digital Fieldscout SC 900 (Spectrum Technologies, Aurora, IL), para lo cual se tomaron muestras hasta una profundidad de $25 \mathrm{~cm}$, cada $2,5 \mathrm{~cm}$, con lo cual se obtuvo un total de 11 datos, los cuales fueron medidos en cuatro épocas: época 1 (E1): antes de la preparación del suelo; época 2 (E2): se realizó en el momento de la preparación del suelo para determinar si se presentaba algún cambio en las propiedades físicas con la utilización de los aperos en la labranza; época 3 (E3): se llevó a cabo a los 100 d después de la siembra, con el fin de observar el cambio en las variables evaluadas a lo largo del tiempo y el efecto del crecimiento longitudinal de las raíces en las propiedades físicas; época 4 (E4): se realizó a los 185 d después de la siembra.

El diámetro ponderado medio (DPM) se determinó con el tamizado en seco el cual proporciona una medida del porcentaje de los diferentes tamaños de agregados, para lo cual se tomó una muestra de 120 g de suelo, se depositó en la torre de tamices, y se colocó en la máquina agitadora durante 10 min, manteniendo constante el tiempo y la velocidad para todas las muestras; luego se determinó la masa de suelo retenida en cada uno de los tamices en una balanza electrónica Acculab VIC 612 de 0,01g de precisión (Sartorius España, Madrid). Para calcular el DPM se utilizó la siguiente fórmula:

$D P M=\sum \bar{X}_{i} \frac{w_{i}}{100}$

En donde: DPM: diámetro ponderado medio; $\bar{X}_{i}$ : diámetro promedio de la fracción para cada tamiz; Wi: porcentaje del peso total de la fracción retenida en cada tamiz. 
Tabla 1. Descripción taxonómica y morfológica del suelo de estudio.

\begin{tabular}{|c|c|c|}
\hline \multicolumn{2}{|l|}{ Taxonomía } & Typic Dystrudepts \\
\hline \multicolumn{2}{|c|}{ Localización } & Municipio de Boyacá (Boyacá) \\
\hline \multicolumn{2}{|l|}{ Sitio } & Finca la Huerta, vía vereda Huerta Grande \\
\hline \multicolumn{2}{|l|}{ Altitud } & 2400 msnm \\
\hline \multicolumn{2}{|c|}{ Ubicación geográfica } & $5^{0} 11^{\prime} 40^{\prime \prime} \mathrm{N}$ y $73^{\circ} 08^{\prime} 44^{\prime \prime} \mathrm{W}$ \\
\hline \multicolumn{2}{|l|}{ Paisaje } & Montaña \\
\hline \multicolumn{2}{|c|}{ Tipo de relieve } & Lomas \\
\hline \multicolumn{2}{|c|}{ Litología o material parental } & Shale gris oscuro con intercalaciones de areniscas y lutitas \\
\hline \multicolumn{2}{|l|}{ Relieve } & Medianamente ondulado \\
\hline \multicolumn{2}{|c|}{ Longitud de la pendiente } & Corta \\
\hline \multicolumn{2}{|c|}{ Gradiente de la pendiente } & $3-5 \%$ \\
\hline \multicolumn{2}{|c|}{ Forma de la pendiente } & Irregular \\
\hline \multicolumn{2}{|c|}{ Clima ambiental } & Frío transicional a seco \\
\hline \multicolumn{2}{|c|}{ Zona de vida } & Bosque húmedo montano bajo (bh-MB) \\
\hline \multicolumn{2}{|c|}{ Clima edáfico } & Údico \\
\hline \multicolumn{2}{|l|}{ Erosión } & Por escurrimiento difuso \\
\hline \multicolumn{2}{|l|}{ Drenaje } & Interno: lento, Externo: moderado; natural, bien drenado \\
\hline \multicolumn{2}{|c|}{ Profundidad efectiva } & Clase: superficial \\
\hline \multicolumn{2}{|c|}{ Limitante de la profundidad } & Cambio textural abrupto \\
\hline \multicolumn{2}{|c|}{ Características diagnósticas } & $\begin{array}{l}\text { Epipedón: Ócrico Endopedón: Cámbico, régimen de humedad údico y baja } \\
\text { saturación de bases. }\end{array}$ \\
\hline \multicolumn{2}{|l|}{ Uso actual } & Cultivos de pastos dedicados a la ganadería bovina \\
\hline \multicolumn{2}{|c|}{ Limitantes del uso } & Fertilidad y precipitación baja \\
\hline \multicolumn{2}{|c|}{ Vegetación natural } & Kikuyo, \\
\hline \multicolumn{2}{|c|}{ Clasificación por capacidad de uso } & Clase: IV \\
\hline \multicolumn{2}{|c|}{ Descrito por } & Yomaira Liney Pinto-Acero \\
\hline $\begin{array}{l}0-20 \mathrm{~cm} \\
\text { Ap }\end{array}$ & \multicolumn{2}{|c|}{$\begin{array}{l}\text { Color en húmedo gris claro (10 YR 6/8), color en seco (7,5 YR 4/3) textura franco arcillosa (FAr), estructura: } \\
\text { bloques angulares. Clase: medianos y gruesos, fuertes, consistencia en húmedo friable en seco dura, en mojado } \\
\text { pegajosa y plástica. Con pocas raíces medias, y poca actividad de microorganismos, límite liso y abrupto; pH 7,6, } \\
\text { ligeramente alcalino. }\end{array}$} \\
\hline $\begin{array}{l}20-37 \mathrm{~cm} \\
\mathrm{Bt}\end{array}$ & \multicolumn{2}{|c|}{$\begin{array}{l}\text { Color en húmedo pardo muy pálido ( } 10 \text { YR } 7 / 3 \text { ), color en seco (2.5 Y 6/6), textura franco arcillosa (FAr), estructura: } \\
\text { bloques angulares y subangulares, Clase: gruesa, fuerte, consistencia en seco dura, en húmedo muy pegajosa, en } \\
\text { mojado plástica; con baja presencia de macroorganismos y baja presencia de raíces, límite claro y ondulado; pH } \\
6,5 \text {, reacción ligeramente ácido. }\end{array}$} \\
\hline $\begin{array}{l}37-70 \mathrm{~cm} \\
\mathrm{BC}\end{array}$ & \multicolumn{2}{|c|}{$\begin{array}{l}\text { Color en húmedo amarillo parduzco (10 YR 6/8), color en seco amarillo (10 YR 7/8), textura: arcillosa (Ar), } \\
\text { estructura: bloques subangulares, Clase: gruesa y fuerte, consistencia en seco dura, en húmedo muy pegajosa; } \\
\text { en mojado plástica; baja porosidad, baja presencia de macroorganismos, y no hay presencia de raíces; pH 6,9, } \\
\text { reacción ligeramente ácido. }\end{array}$} \\
\hline
\end{tabular}

La distribución por tamaño de agregados se determinó como el porcentaje de tamaño medio de agregados vs. el porcentaje acumulado de agregados.
Con los datos, se realizó pruebas de normalidad y homogeneidad de varianzas, posteriormente se hicieron análisis de varianza para determinar las diferencias estadísticas con un grado de confiabi- 
lidad del 95\%, y así determinar los mejores tratamientos con pruebas de comparación de promedios Tukey $(P \leq 0,05)$. Los análisis se realizaron con el programa $\mathrm{R}$ versión 3.1.2.2.

\section{RESULTADOS Y DISCUSIÓN}

Antes de la preparación del suelo (E1), el análisis de varianza no mostró diferencias estadísticas significativas entre tratamientos ni bloques (tabla 2), lo que significa que el suelo donde se desarrolló el estudio era estadísticamente homogéneo. Se observó que la resistencia a la penetración es cada vez mayor a medida que se profundiza en el perfil del suelo, independientemente del tipo de labranza empleado, aunque el contenido de humedad también influye en la compactación (Kirsch, 2010), caso contrario a lo reportado por Henríquez et al. (2011), quien en un suelo clasificado como Dystrudepts de Costa Rica usado en cafetales, determinaron que el nivel de compactación diferencial, en la superficie del suelo mostró los mayores valores de las variables relacionadas a la compactación que los valores encontrados a los $15 \mathrm{~cm}$. Los valores de la E1 coinciden con lo reportado por Siczek y Lipiec (2011), quienes afirman que la compactación inicial antes de cualquier arado está asociada al paso del tractor.

En el muestreo realizado después de la preparación del suelo (E2), no se presentó diferencias estadísticas entre tratamientos, pero sí entre bloques (tabla 1), donde la LM hasta los 25 $\mathrm{cm}$ de muestreo presentó los valores más altos de resistencia a la penetración, lo cual coincide con lo reportado por Keller et al. (2013), quienes mencionan que altos valores de compactación reducen el volumen de poros disponibles para el almacenamiento de fluidos. El suelo preparado con LC y LV presentó la menor resistencia a la penetración, debido a que la mecanización conlleva a la generación de nuevos macroporos estructurales (Cortés et al., 2013). Para el caso de los tres bloques, se presentó diferencias altamen- te significativas en la E2 a los 12,5; 17,5 y $20 \mathrm{~cm}$, para la LV con valores de menor resistencia a la penetración de 262,8; 810,8 y $1337,5 \mathrm{kPa}$, respectivamente, comparado con la LM, la cual tuvo mayor resistencia con valores de 665, 1.192,4 y $1.775,9 \mathrm{kPa}$, respectivamente, y LC con valores intermedios de 497,9; 1.000,2 y $1.566 \mathrm{kPa}$ respectivamente (figura $1 \mathrm{~B}$ ).

En cuanto a la E3, se evidenciaron diferencias altamente significativas a los 12,5 y $20 \mathrm{~cm}$ de profundidad, en donde la LV con valores promedio de $312,8 \mathrm{kPa}$, en comparación con la LM y LC con valores de 685 y $557,9 \mathrm{kPa}$ respectivamente (figura 1C). Para la última época de muestreo (E4), se presentaron diferencias altamente significativas a los $20 \mathrm{~cm}$ de profundidad, en donde la LV tuvo valores promedio de 1.347,5 kPa, comparándola con la LM y LC $1.885,9$ y $1.716 \mathrm{kPa}$, respectivamente (figura $1 \mathrm{D}$ ).

Posiblemente, como las mediciones se realizaron después de ser preparado el suelo, los aperos utilizados en LV y LC permitieron la ruptura de los agregados del suelo y por ende disminuyó los valores de presión ejercidos en el perfil. Al respecto, Lipiec et al. (2012) afirman que al aumentar la compactación aumentan los agregados de tamaño $>3 \mathrm{~mm}$, lo que coincide con los resultados obtenidos en este trabajo. Sin embargo, es importante tener en cuenta que a medida que se profundiza en el perfil del suelo este tiende a ser más compacto, probablemente, una de las causas sea el tráfico de la maquinaria agrícola, cuyo efecto es acumulativo, así como a la carga in situ producida por los neumáticos agrícolas (Keller y Lamandé, 2010), los diferentes tipos de labranza (Kim et al., 2010), y las condiciones iniciales del suelo (Lamandé y Schjonning, 2011). Botta et al. (2002) consideran que es aconsejable dividir la compactación del suelo en dos: compactación superficial y compactación del subsuelo, ya que la compactación del subsuelo está directamente influenciada por el peso total del rodado (Alaouit et al., 2011), siendo independiente de la presión ejercida por el contacto rueda-suelo, establecien- 
Tabla 2. P-valor de la resistencia a la penetración con tres tipos de labranza en un Inceptisol en Boyacá-Boyacá a diferentes profundidades.

\begin{tabular}{|c|c|c|c|c|c|}
\hline Profundidad (cm) & Época & Labranza vertical & Labranza mínima & $\begin{array}{c}\text { Labranza } \\
\text { convencional }\end{array}$ & P-valor \\
\hline \multirow{4}{*}{0} & E1 & 110,0 a & 105,0 a & 115,0 a & $0,631 \mathrm{~ns}$ \\
\hline & E2 & $44,7 \mathrm{a}$ & $101,7 \mathrm{~b}$ & $58,3 \mathrm{ab}$ & $0,044^{*}$ \\
\hline & E3 & 74,7 a & $102,7 \mathrm{~b}$ & $68,3 a b$ & $0,049 *$ \\
\hline & E4 & $71,7 \mathrm{a}$ & $101,0 \mathrm{~b}$ & $61,3 \mathrm{ab}$ & $0,039 *$ \\
\hline \multirow{4}{*}{2,5} & E1 & 140,0 a & 125,0 a & 130,0 a & $0,674 \mathrm{~ns}$ \\
\hline & E2 & 58,3 a & $125,0 \mathrm{~b}$ & $89,2 a b$ & $0,049 *$ \\
\hline & E3 & 88,3 a & $121,0 \mathrm{~b}$ & $109,2 \mathrm{ab}$ & $0,029 *$ \\
\hline & E4 & 81,3 a & $118,0 \mathrm{~b}$ & $102,2 \mathrm{ab}$ & 0,048 * \\
\hline \multirow{4}{*}{5} & E1 & 210,0 a & 245,0 a & 225,0 a & $0,693 \mathrm{~ns}$ \\
\hline & E2 & $103,1 \mathrm{a}$ & $212,2 \mathrm{~b}$ & $159,2 \mathrm{ab}$ & $0,041 *$ \\
\hline & E3 & $123,1 \mathrm{a}$ & $216,2 \mathrm{~b}$ & $179,2 \mathrm{ab}$ & $0,031 *$ \\
\hline & E4 & $113,1 \mathrm{a}$ & $218,2 \mathrm{~b}$ & $171,2 \mathrm{ab}$ & $0,049 *$ \\
\hline \multirow{4}{*}{7,5} & E1 & 340,0 a & 380,0 a & 365,0 a & $0,513 \mathrm{~ns}$ \\
\hline & E2 & 134,2 a & 326,6 a & $271,7 \mathrm{ab}$ & $0,039 *$ \\
\hline & E3 & $164,2 \mathrm{a}$ & $336,6 \mathrm{~b}$ & 291,7 b & $0,036^{*}$ \\
\hline & E4 & $151,2 \mathrm{a}$ & $334,6 \mathrm{~b}$ & $281,7 \mathrm{ab}$ & $0,019 *$ \\
\hline \multirow{4}{*}{10} & E1 & 630,0 a & 650,0 a & 675,0 a & $0,493 \mathrm{~ns}$ \\
\hline & E2 & 212,3 a & $529,8 \mathrm{~b}$ & $391,4 \mathrm{ab}$ & $0,032 *$ \\
\hline & E3 & 272,3 a & $539,8 \mathrm{~b}$ & $421,4 \mathrm{~b}$ & $0,012 *$ \\
\hline & E4 & 252,3 a & $531,8 \mathrm{~b}$ & $411,4 b$ & $0,023 *$ \\
\hline \multirow{4}{*}{12,5} & E1 & 810,0 a & 790,0 a & 835,0 a & $0,623 \mathrm{~ns}$ \\
\hline & E2 & 262,8 a & $665,0 \mathrm{~b}$ & $497,9 \mathrm{~b}$ & $0,0016^{* *}$ \\
\hline & E3 & 312,8 a & $685,0 \mathrm{~b}$ & $557,9 \mathrm{~b}$ & $0,0086 * *$ \\
\hline & E4 & 302,8 a & $694,0 \mathrm{~b}$ & $567,9 \mathrm{~b}$ & 0,036 * \\
\hline \multirow{4}{*}{15} & E1 & 890,0 a & 950,0 a & 930,0 a & $0,423 \mathrm{NS}$ \\
\hline & E2 & 372,8 a & 798,2 b & $640,7 \mathrm{~b}$ & $0,038 *$ \\
\hline & E3 & 472,8 a & $828,2 \mathrm{~b}$ & $690,7 \mathrm{~b}$ & $0,021 *$ \\
\hline & E4 & 452,8 a & $833,2 \mathrm{~b}$ & $698,7 \mathrm{ab}$ & $0,047 *$ \\
\hline \multirow{4}{*}{17,5} & E1 & $1.210,0 \mathrm{a}$ & $1.330,0 \mathrm{a}$ & $1.270,0 \mathrm{a}$ & $0,393 \mathrm{~ns}$ \\
\hline & E2 & 810,8 a & $1.192,4 \mathrm{~b}$ & $1.000,2 \mathrm{~b}$ & 0,0021 \\
\hline & E3 & 910,8 a & $1.232,4 b$ & $1.100,2 a b$ & $0,045^{*}$ \\
\hline & E4 & 880,8 a & $1.222,4 \mathrm{~b}$ & $1.140,2 a b$ & $0,049 *$ \\
\hline \multirow{4}{*}{20} & E1 & $1.990,0 \mathrm{a}$ & $1.910,0 \mathrm{a}$ & $1.960,0 \mathrm{a}$ & $0,335 \mathrm{~ns}$ \\
\hline & E2 & $1.337,5 \mathrm{a}$ & $1.775,9 \mathrm{~b}$ & $1.566,0 \mathrm{~b}$ & $0,0025 * *$ \\
\hline & E3 & $1.337,5 \mathrm{a}$ & $1.845,9 \mathrm{~b}$ & $1.696,0 \mathrm{~b}$ & 0,0056 ** \\
\hline & E4 & $1.347,5 \mathrm{a}$ & $1.885,9 \mathrm{~b}$ & $1.716,0 \mathrm{~b}$ & $0,0013^{* *}$ \\
\hline \multirow{4}{*}{22,5} & E1 & $2.400,0 \mathrm{a}$ & $2.460,0 \mathrm{a}$ & $2.350,0 \mathrm{a}$ & $0,403 \mathrm{~ns}$ \\
\hline & E2 & $2.183,9 \mathrm{a}$ & $2.390,2 a b$ & $2.334,7 a b$ & $0,042 *$ \\
\hline & E3 & $2.233,9 \mathrm{a}$ & $2.430,2 \mathrm{~b}$ & $2.354,7 \mathrm{~b}$ & $0,034^{*}$ \\
\hline & E4 & $2.263,9 \mathrm{a}$ & $2.490,2 \mathrm{~b}$ & $2.394,7 a b$ & $0,046 *$ \\
\hline \multirow{4}{*}{25} & E1 & $2.580,0 \mathrm{a}$ & $2.600,0 \mathrm{a}$ & $2.550,0 \mathrm{a}$ & $0,583 \mathrm{~ns}$ \\
\hline & E2 & $2.395,2 \mathrm{a}$ & $2.508,8 \mathrm{a}$ & $2.467,6 \mathrm{a}$ & $0,078 \mathrm{~ns}$ \\
\hline & E3 & $2.435,2 \mathrm{a}$ & $2.518,8 \mathrm{a}$ & $2.497,6 \mathrm{a}$ & $0,052 \mathrm{~ns}$ \\
\hline & E4 & $2.485,2 \mathrm{a}$ & $2.558,8 \mathrm{a}$ & $2.527,6 \mathrm{a}$ & $0,097 \mathrm{~ns}$ \\
\hline
\end{tabular}

**Diferencias significativas al $1 \%$ según la prueba de Tukey; *Diferencias significativas al $5 \%$; ns: no hay diferencias, de acuerdo con el análisis de varianza. E1: antes de la preparación del suelo; E2: después de la preparación del suelo; E3: 100 d después de la siembra; E4: 185 d después de la siembra 
do que, a mayor peso, mayor es la profundidad a la que se espera encontrar la compactación.

La degradación física del suelo tiene como causa principal la compactación (Pagliai et al., 2003). Al respecto, Terminiello et al. (2007) y Hamza y Anderson (2005) resaltan la necesidad de reducir el peso total del conjunto tractor-implemento, pero se debe tener en cuenta que esto implica utilizar equipos más pequeños y por tanto la necesidad de aumentar el número de pasadas sobre cada hectárea de terreno a trabajar, lo cual puede causar mayor o igual compactación que con equipos de mayor magnitud. Caso contrario a lo reportado por Kirby et al. (1997) quienes afirman que, en suelo seco, las tensiones en los primeros $10 \mathrm{~cm}$ de profundidad son transmitidas principalmente a través de los panes del neumático, los cuales ocupan alrededor de un tercio del área de contacto; sin embargo, a mayor profundidad este efecto no fue significativo.

Forsythe et al. (2005) mencionan que se encuentra una mayor resistencia en los primeros centímetros del suelo bajo siembra directa y labranza mínima con respecto a labranza convencional. Posiblemente en la E3 la adición de agua debido a la precipitación incrementó la cohesión, y originó un efecto de lubricación entre las partículas, el cual permitió que estas fuesen realineadas más fácilmente durante el proceso de compresión, donde el aire pudo ser desalojado de los poros, favoreciendo la compactación (González et al., 2008).

\section{Estabilidad estructural}

En la tabla 3 se puede observar que la estabilidad estructural medida a través del DPM no presentó diferencias estadísticas significativas entre tratamientos, pero sí entre bloques. La tendencia general del DPM fue disminuir después de la preparación del suelo y luego aumentar en las épocas 3 y 4 (a los 100 y 185 d después de la preparación) (figura 2).
En la E1 no hubo diferencias estadísticas significativas, con un promedio de $3,96 \mathrm{~mm}$ partiendo de un suelo estadísticamente homogéneo. Después de la preparación del suelo, E2, disminuyeron todos los valores de la DPM, debido a que cambia el orden de los agregados, los cuales son entidades dinámicas y cuya estabilidad depende del tipo de preparación del suelo (García-Orenes et al., 2009), por lo que es probable que esto haya generado las diferencias estadísticas, siendo la LM la única considerada dentro de un rango estable con un valor promedio de $3,177 \mathrm{~mm}$, posiblemente a que no hubo ruptura del suelo (Baumgarten et al., 2012), y que la fuerza de atracción de Van der Walls se mantuvo (Zhang et al., 2012; Yu et al., 2012), y donde las LV y LC presentaron valores promedio de 2,864 y 2,655 mm respectivamente, considerados moderadamente estables (IGAC, 2006). Dexter y Czyz (2011) afirman que los agregados en el rango de 5 a $3 \mathrm{~mm}$ son aptos para la emergencia de las plantas, y que la presencia de macroagregados posibilita una fácil accesibilidad a la biomasa microbiana (Awad et al., 2013), parte importante para mantener la estabilidad de agregados a largo plazo (Gentile et al., 2011).

A los 100 días después de preparación del suelo (E3) no se presentó diferencias estadísticas, pero aumentaron los valores en esta evaluación, debido a que el DPM por lo general tiende a aumentar con el tiempo a causa de la incorporación de sustancias orgánicas y exudaciones radiculares (Grosbellet et al., 2011). La LM y LV presentaron valores de DPM acordes a los rangos del IGAC (2006), los cuales se consideran estables con valores promedios de 3,194 y 3,001 mm respectivamente, frente a la LC con un valor promedio de $2,712 \mathrm{~mm}$, moderadamente estable.

A los 185 ddps (E4) se presentaron diferencias estadísticas significativas, donde LM y LV continuaron con valores estables de DPM mostrando promedios de 3,201 y 3,093 mm respectivamente, en comparación con LC que presentó un 


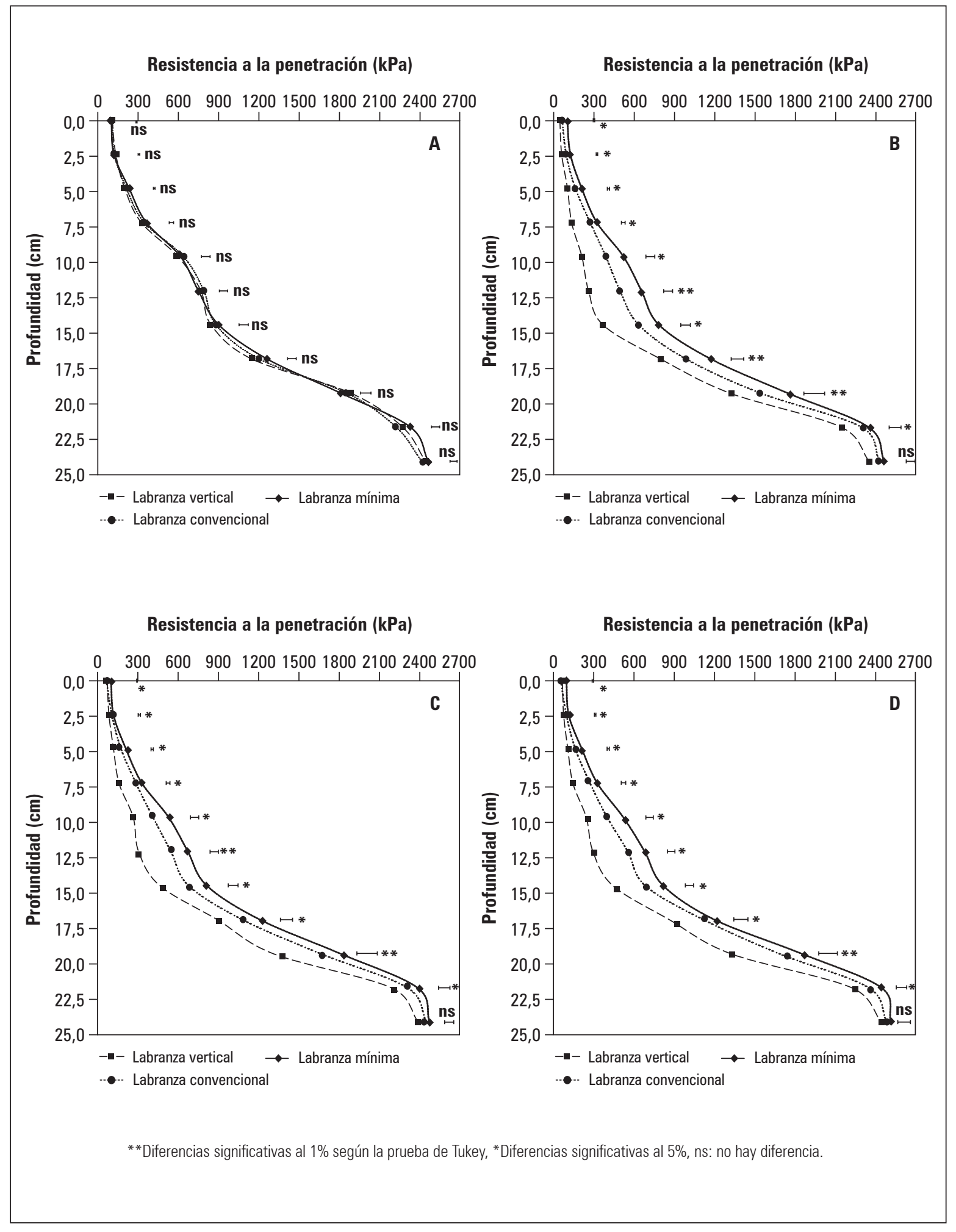

Figura 1. Comportamiento de la resistencia a la penetración para los diferentes tipos de labranza. A) Antes de la preparación del suelo; B) Después de la preparación del suelo; C) A los 100 DPS y D) 185 DPS. Las barras horizontales indican el error estándar $(n=18)$. 


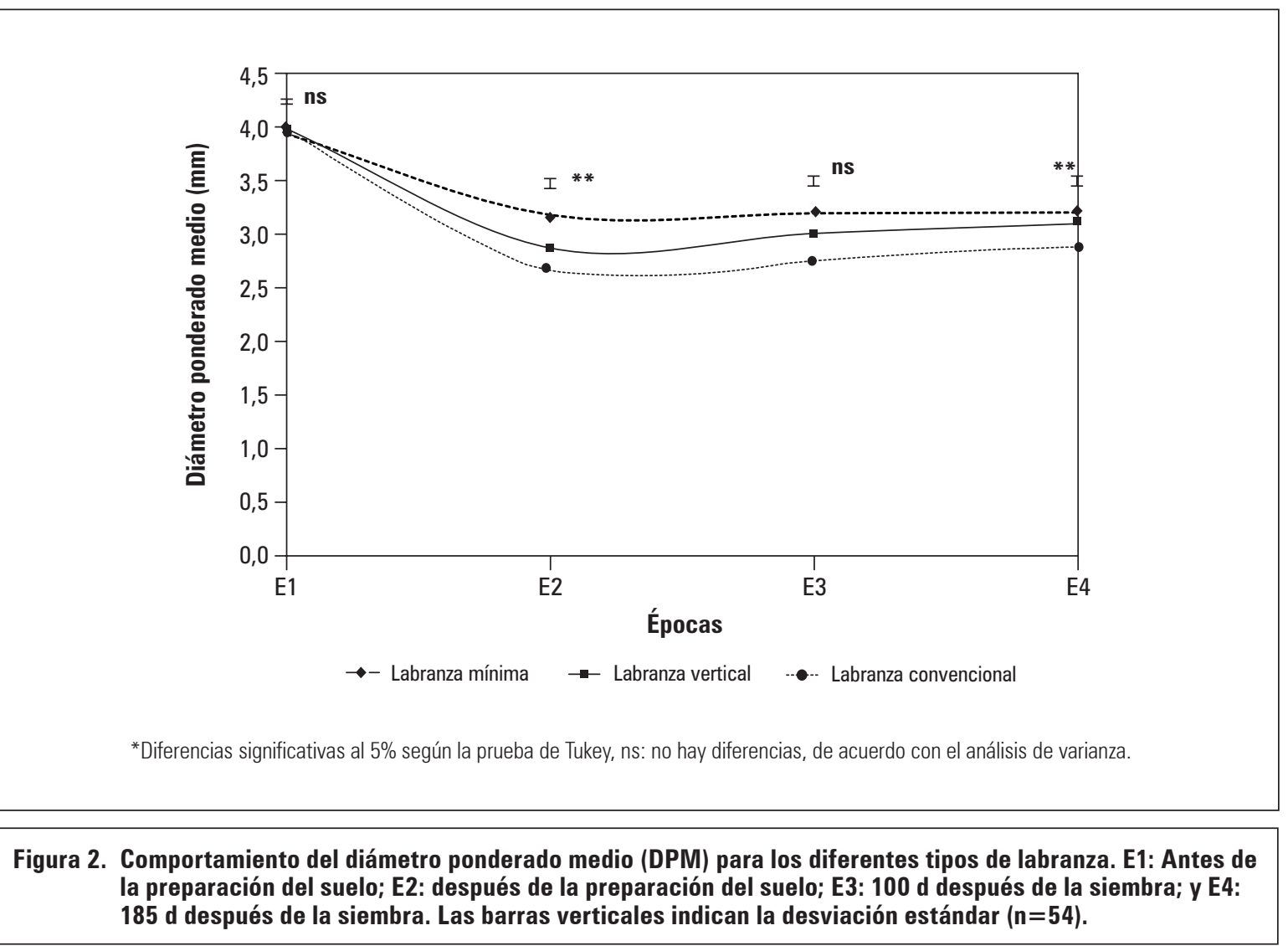

DPM de 2,86 considerado moderadamente estable (figura 2), lo que destaca el papel del tipo de labranza en la reconfiguración de las partículas del suelo y la incidencia en el crecimiento de la arracacha y su relación con la distribución y el tamaño de los agregados.

Según Lal y Shukla (2004) existen diversos factores que promueven la formación de agregados, uno de ellos es la presencia de arcillas, las cuales favorecen la agregación, situación que pudo originar el aumento del DPM a lo largo del tiempo. Además la LM mostró los valores más altos de DPM para todas las épocas después de la preparación del suelo (E2, E3 y E4) con valores promedio de 3,$17 ; 3,19$ y 3,20 respectivamente, lo que se debe probablemente a que durante la aplicación de cargas, las partículas del suelo son reorganizadas, decrece el espacio poroso, lo cual incrementa la densidad, cambia la forma, el tamaño y la distribución de los poros, limita la capacidad de retención de humedad y nutrientes del suelo, el intercambio hídrico y gaseoso, y aumenta la impedancia mecánica (Gysi et al., 2001).

Para el porcentaje acumulado de agregados en la primera evaluación antes de la preparación, se parte de un suelo estadísticamente homogéneo, donde el promedio de agregados con un diámetro superior a $6 \mathrm{~mm}$ fue del $57,61 \%$, y entre los agregados de 1,5 y $3 \mathrm{~mm}$ hay un promedio de $13,11 \%$; se observan agregados con porcentajes inferiores a $0,375 \mathrm{~mm}$ como los menos ideales en un suelo con un promedio de $5,56 \%$, teniendo en cuenta que una proporción alta de microagregados se puede considerar como indicador de degradación estructural del suelo (Baumgarten et al., 2012). De igual manera, si hay un mayor número de agregados entre 1 y $3 \mathrm{~mm}$ que estén distribuidos en forma tal que la capa no sea completamente homogénea, las relaciones de intercambio gaseoso son mejores y el suelo puede ad- 
Tabla 3. P-valor y promedios del diámetro ponderado medio (DPM) con tres tipos de labranza en un Inceptisol en Boyacá-Boyacá en cuatro épocas de evaluación.

\begin{tabular}{|c|c|c|c|c|}
\hline Época & Labranza vertical & Labranza mínima & Labranza convencional & $p$-valor \\
\hline E1 & $3,991 \mathrm{a}$ & $3,9386 \mathrm{a}$ & $3,9558 \mathrm{a}$ & $0,680 \mathrm{~ns}$ \\
\hline E2 & $2,864 \mathrm{a}$ & $3,1779 \mathrm{~b}$ & $2,6551 \mathrm{ab}$ & $0,047^{*}$ \\
\hline E3 & $3,001 \mathrm{a}$ & $3,1940 \mathrm{a}$ & $2,7121 \mathrm{a}$ & $0,053 \mathrm{~ns}$ \\
\hline E4 & $3,093 \mathrm{a}$ & $3,2015 \mathrm{a}$ & $2,8600 \mathrm{a}$ & $0,049{ }^{*}$ \\
\hline
\end{tabular}

quirir y conservar mayor cantidad de agua (Lal y Shukla, 2004).

Después de la preparación del suelo (E2) no hubo diferencias estadísticas con los agregados de tamaño mayores a $6 \mathrm{~mm}(33,4 \%)$, estableciéndose una reducción de un $24,21 \%$ en relación con la E1. Sin embargo, para los agregados de tamaño entre 3 y $6 \mathrm{~mm}$ sí hubo diferencias estadísticas significativas, se observa en la figura $3 \mathrm{~B}$, que el mayor porcentaje lo presenta la LV con un valor promedio de $29,4 \%$, frente a LC y LM con valores de $19 \%$ y $21,2 \%$ respectivamente. Para el caso de LV, se considera un suelo estable, debido a que presenta el mayor número de agregados por encima de los $2 \mathrm{~mm}$, respecto a la clasificación dada por Volveras y Amézquita (2009).

Para los agregados con diámetro entre 1,5 y 3 $\mathrm{mm}$ no hubo diferencias estadísticas significativas, ya que los tres tipos de labranza tienen un promedio de $13,56 \%$ de agregados. Para el tamaño de agregados comprendido entre $0,75 \mathrm{~mm}$ y 1,5 $\mathrm{mm}$ hubo diferencias estadísticas significativas, donde la LC tiene el valor más alto con un promedio de $11 \%$ de agregados, frente a la LV y LM con valores de $3,36 \%$ y $5,51 \%$ respectivamente. Esto probablemente se debe a que existió una modificación principalmente del tamaño de los agregados asociados a la baja estabilidad o resistencia de los mismos.

Por último, para los agregados comprendidos entre el rango de 0,375 y $0,75 \mathrm{~mm}$ se observa- ron diferencias estadísticas significativas, donde nuevamente y para los diámetros inferiores los más altos porcentajes de agregados los tiene la LC frente a la LV y LM, teniendo la LV los menores valores de los agregados de estos diámetros.

A los 100 ddps (E3) no se presentaron diferencias estadísticas en los agregados de tamaño mayores a $6 \mathrm{~mm}$ con un valor promedio de 40,4\%, aumentando en promedio $7 \%$ los agregados de este diámetro en relación a la E2, esto pudo ocurrir debido a que el suelo presenta altos contenido de calcio $\left(\mathrm{Ca}^{2+}\right)$, lo cual favorece la estructura, y también a que los cationes absorbidos y el manejo del suelo influyen en este proceso (Ferreira et al., 2000).

Adicionalmente, los agregados entre 3 y $6 \mathrm{~mm}$ mostraron diferencias significativas, ya que el mayor porcentaje lo reportó la LV con un valor promedio de $31,4 \%$, frente a LC y LM con valores de $22 \%$ y $25,2 \%$ respectivamente, obteniéndose en general un aumento en los tres tipos de labranza, pero en mayor proporción la LM. No obstante, para los agregados con diámetro de entre 1,5 y $3 \mathrm{~mm}$ no hubo diferencias estadísticas significativas, pero se pudo observar (figura 3C) que en este diámetro el valor promedio de la LM y LC es un $50 \%$ menor que al de la LV. Para el diámetro de agregados entre 0,75 mm y $1,5 \mathrm{~mm}$ no hay diferencias significativas y las tres labranzas presentaron un valor de 7,95\% de agregados en este rango; para los diámetros inferiores a $0,75 \mathrm{~mm}$ se evidenciaron diferencias estadísticas donde la LC posee los porcentajes 

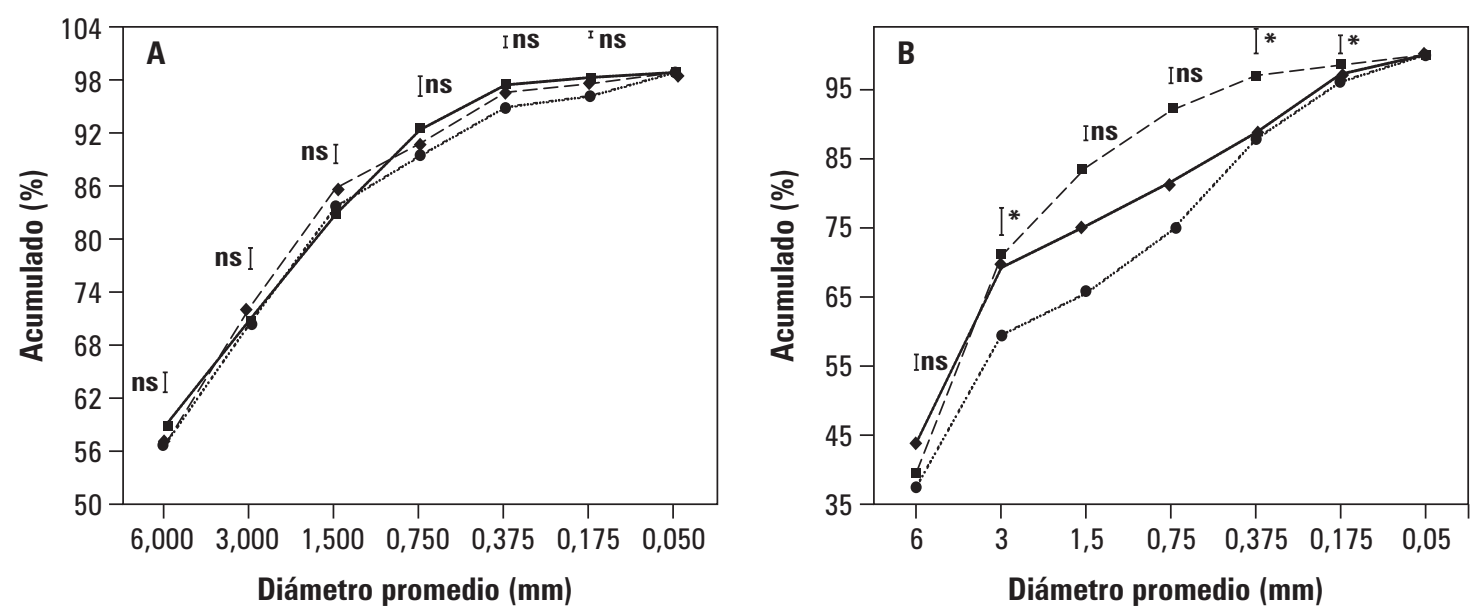

-- Labranza vertical $\rightarrow$ Labranza mínima

-- Labranza convencional

$\rightarrow-$ Labranza vertical $\rightarrow$ Labranza mínima

-- Labranza convencional
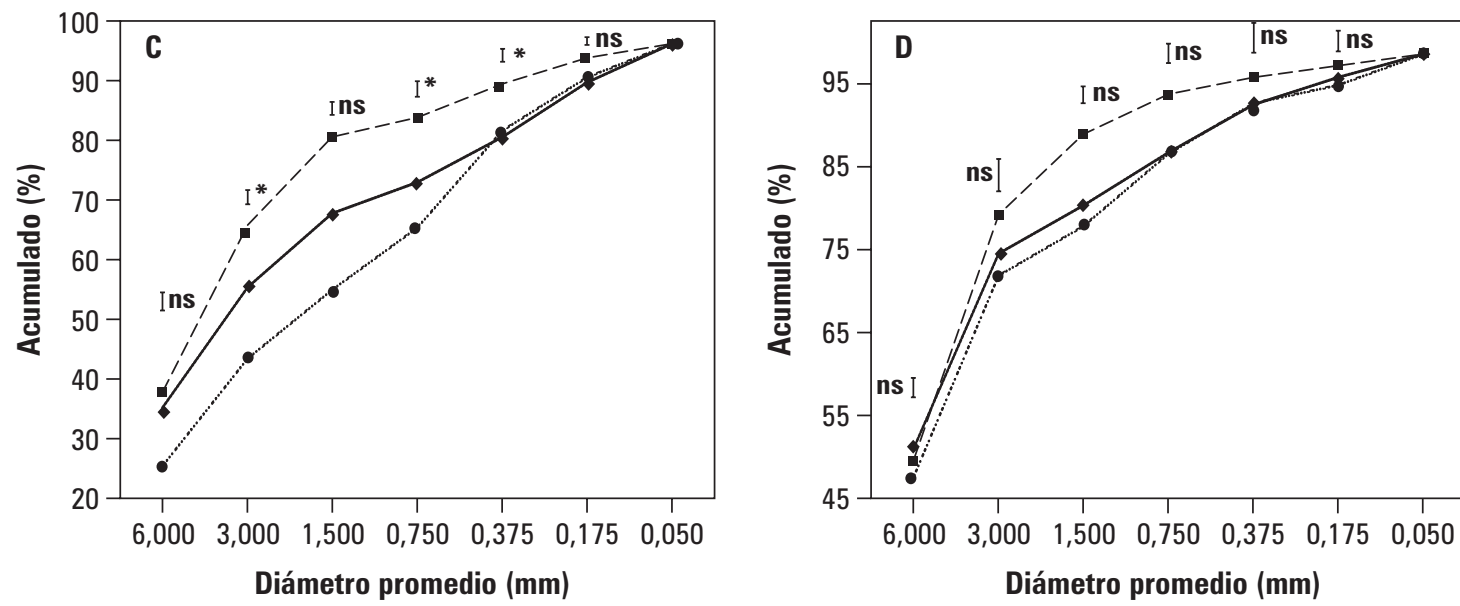

-- Labranza vertical $\longrightarrow$ Labranza mínima

-.-. Labranza convencional

- - Labranza vertical $\rightarrow$ Labranza mínima

-.- Labranza convencional

*Diferencias significativas al 5\% según la prueba de Tukey, ns: no hay diferencias, de acuerdo con el análisis de varianza.

Figura 3. Comportamiento de la distribución por tamaño de agregados para los diferentes tipos de labranza. A) Antes de la preparación del suelo (E1); B) Después de la preparación del suelo (E2); C) 100 d después de la siembra (E3); D) 185 d después de la siembra (E4). Las barras verticales indican la desviación estándar (n=18).

más altos de agregados en estos diámetros, lo cual coincide con los estudios reportados por Zhang et al. (2012), quienes mencionan que la LC fue la que más generó la reducción de agregados y estabilidad estructural. Por lo anterior, se pudo observar un proceso de degradación estructural del suelo en el tiempo después de la siembra, con altos porcentajes de agregados de diámetro menor a $2 \mathrm{~mm}$, coincidiendo con reportes de Kim et al. (2010) quienes afirman 
que una proporción alta de microagregados se puede considerar como indicador de degradación estructural del suelo.

A los 185 ddps (E4) no se presentaron diferencias estadísticas en los agregados para ninguno de los tamaños, sin embargo, para los agregados mayores a $6 \mathrm{~mm}$ con un valor promedio de $49,4 \%$, se presentó un aumento, en un promedio de $9 \%$ y en relación a la E1, un 8,2\% menos. Lo anterior se manifestó a través del tiempo y después de la preparación del suelo, en donde este tiende a recuperar su capacidad de agregación, es decir, probablemente tiende a presentar resiliencia estructural dependiendo del manejo, sin embargo, se considera que el tipo de labranza influye en los procesos de agregación según su tamaño, mas no de forma, y tiene una estrecha relación con el crecimiento radicular de la arracacha, caso similar a lo mencionado por Feinan et al. (2015) quienes manifiestan que cuando se presentan microgrietas, estas pueden ser estabilizadas por las acciones de crecimiento de las raíces que las atraviesan.

\section{CONCLUSIONES}

El tipo de labranza ejerce una influencia directa en la resistencia a la penetración y estabilidad estructural, medida a través del DPM y distribución de agregados por tamaño después de la preparación del suelo y hasta 185 días después de la preparación del suelo.

La labranza vertical (LV) presentó la mejor estabilidad estructural en un Typic Dystrudepts, franco arcilloso; con un mayor número de agregados entre 1 y $3 \mathrm{~mm}$, lo cual permite que el suelo pueda adquirir y conservar mayor cantidad de agua en relación con la labranza convencional (LC).

Los tres tipos de labranza tienen una profundidad de acción de $20 \mathrm{~cm}$ en promedio, en donde la LV a lo largo del tiempo presenta los valores más bajos de resistencia a la penetración debido a que tiende a descompactar el suelo más fácilmente.

\section{AGRADECIMIENTOS}

Los autores agradecen para el desarrollo del proyecto de investigación a la Corporación PBA, con el apoyo de Colciencias. Así mismo agradecen a la Dirección de Investigaciones de la Universidad Pedagógica y Tecnológica de Colombia, por el financiamiento a través del proyecto externo SGI 1778.

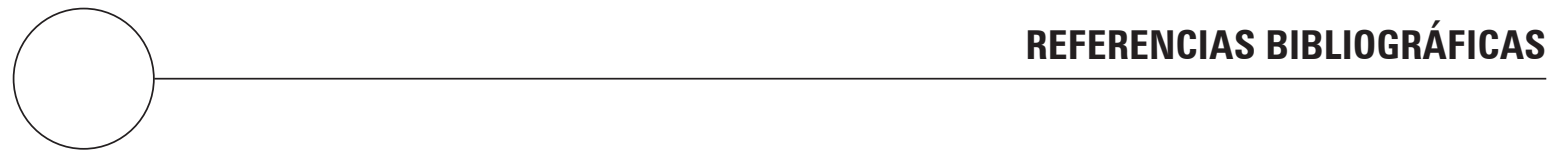

Alaouit, A., J. Lipiec y H.H. Gerke. 2011. A review of the changes in the soil pore system due to soil deformation: a hydrodynamic perspective. Soil Tillage Res. 115-116, 1-15.

Alvarado, A. y L. Ochoa. Tecnologías locales de producción de arracacha (Arracacia xanthorrhiza Bancroft) en el municipio de Boyacá, departamento de Boyacá. Rev. UDCA Act. Div. Cient. 13(1), 125-133.

Awad, Y.M., E. Blagodatskaya, Y.S. Ok y Y. Kuzyakov. 2013. Effects of polyacrylamide, biopolymer and biochar on the decomposition of 14C-labelled maize residues and on their stabilization in soil aggregates. Eur. J. Soil Sci. 64, 488-499. Doi: 10.1111/ ejss.12034

Barthes, B. y E. Roose. 2002. Aggregate stability as an indicator of soil susceptibility to runoff and erosion; validation at several levels. Catena 47, 133 149. Doi: 10.1016/S0341-8162(01)00180-1

Baumgarten, W., T. Neugebauer, E. Fuchs y R. Horn. 2012. Structural stability of Marshland soils of the riparian zone of the tidal Elbe River. Soil Tillage Res. 125, 80-88. Doi: 10.1016/j. still.2012.06.002 
Botta, G., D. Jorajuria y L. Draghi. 2002. Influence of the axle load, tire size and configuration, on the compaction of a freshly tilled clayey soil. J. Terramechanics 39, 47-54. Doi: 10.1016/S00224898(02)00003-4

Casanova, E. y D. Lobo. 2007. Relación entre la física y la fertilidad de los suelos. Venesuelos 15(1), 42-56.

Cortés, C., J. Camacho-Tamayo y F. Leiva. 2013. Análisis multivariado del comportamiento espacial y temporal de la resistencia del suelo a la penetración. Acta Agron. 62(3), 268-278.

Dexter, A.R. y E.A. Czyz. 2011. Soil crumbling during tillage as a function of soil organic matter content. Int. Agrophysics (25), 215-221.

EOT. Esquema de Ordenamiento Territorial del municipio de Boyacá. 2003. Alcaldía Municipal de Boyacá, Boyacá.

Feinan, H., X. Chenyang, L. Hang, L. Song, Y. Zhenghong, L. Yue y H. Xinhua. 2015. Particles interaction forces and their effects on soil aggregates breakdown. Soil Tillage Res. 147, 1-9. Doi: 10.1016/j.still.2014.11.006

Ferreira, A.J.D., C. Coelho, R. Walsh, R.A. Shakesby, A. Ceballos y S. Doerr. 2000. Hydrological implications of soil water-repellency in Eucalyptus globulus forests, north-central Portugal. J. Hydrology 231-232, 165-177.

Forsythe, W., F. Sancho y M. Villatoro. 2005. Efecto de la compactación de los suelos sobre el rendimiento de maíz en tres localidades de Costa Rica. Agron. Costar. 29(3), 175-185.

García-Orenes, F., A. Cerda, J. Mataix-Solera, C. Guerrero, M. Bodi, V. Arcenegui, R. Zornoza y J. Sempere. 2009. Effects of agricultural management on Surface soil propierties and soil-water losses in Eastern Spain. Soil Tillage Res. 106, 117-123.

Gentile, R., B. Vanlauwe y J. Six. 2011. Litter quality impacts short- but not long-term soil carbon dynamics in soil aggregate fractions. Ecol. Applications 21, 695-703. Doi: 10.2307/23021619

González, O., C. Iglesias, M. Herrera, A. López y A. Iznaga. 2008. Efecto de la humedad y la presión sobre el suelo en la porosidad total de un Rhodic Ferralsol. Rev. Cienc. Téc. Agropecu. 17(2), 50-54.

Grosbellet, C., L. Vidal-Beaudet, V. Caubel y S. Charpentier. 2011. Improvement of soil structure formation by degradation of coarse organic matter. Geoderma 162(1), 27-38. Doi: 10.1016/j.geoderma. 2011.01.003
Gysi, M., V. Maeder y P. Weisskopf. 2001. Pressure distribution underneath tires of agricultural vehicles. Transactions of ASA 44(6), 1385-1389. Doi: 10.13031/2013.7001

Hamza M. y W. Anderson W. 2005. Soil compaction in cropping systems: A review of the nature, causes and possible solutions. Soil Tillage Res. 82, 121145. Doi: 10.1016/j.still.2004.08.009

Henríquez, C., O. Ortiz, K. Largaespada, P. Portuguez, M. Vargas, P. Villalobos y D. Gómez. 2011. Determinación de la resistencia a la penetración, al corte tangencial, densidad aparente y temperatura en un suelo cafetalero, Juan Viñas, Costa Rica. Agronomía Costarricense 35(1), 175-184.

IGAC. 2006. Métodos analíticos de laboratorio de suelos. $6^{a}$ ed. Instituto Geográfico Agustín Codazzi, Bogotá.

Keller, T. y M. Lamande. 2010. Challenges in the development of analytical soil compaction models. Soil Tillage Res. 111, 54-64. Doi: 10.1016/j. still.2010.08.004

Keller, T., M. Lamande, S. Peth, M. Berli, J.-Y. Delenne, W. Baumgarten, W. Rabbel, F. Radja1, J. Rajchenbach, A.P.S. Selvadurai y D. Or. 2013. An interdisciplinary approach towards improved understanding of soil deformation during compaction. Soil Tillage Res. 128, 61-80. Doi: 10.1016/j. still.2012.10.004

Kim, H., S.H. Anderson, P.P. Motavalli y C.J. Gantzer. 2010. Compaction effects on soil macropore geometry and related parameters for an arable field. Geoderma 160, 244-251. Doi: 10.1016/j.geoderma.2010.09.030

Kirby, J., B. Blunden y C. Trein. 1997. Simulating soil deformation using a critical state model: II. Soil compaction beneath tyres and tracks. Eur. J. Soil Sci. 48, 59-70. Doi: 10.1111/j.1365-2389.1997.tb00185.x

Kirsch, R. 2010. Petrophysical properties of permeable and low-permeable rocks. pp. 1-22. En: Kirsch, R. (ed.). Groundwater geophysics. $2^{\text {nd }}$ ed. Springer-Verlag, Berlin.

Lamandé, M. y P. Schjonning. 2011. Transmission of vertical stress in a real soil profile. Part III. Effect of soil water content. Soil Tillage Res. 114, 78-85. Doi: 10.1016/j.still.2010.10.001

Lipiec, J. M. Hajnos y R. wieboda. 2012. Estimating effects of compaction on pore size distribution of soil aggregates by mercury porosimeter. Geoderma 179-180, 20-27. doi:10.1016/j.geoderma.2012.02.014 
Pagliai, M., A. Marsili, P. Servadio, N. Vignozzi y S. Pellegrini. 2003. Changes in some physical properties of a clay soil in Central Italy following the passage of rubber tracked and wheeled tractors of medium power. Soil Tillage Res. 73, 119-129. Doi: 10.1016/ S0167-1987(03)00105-3

Ramírez-López, L., A. Reina-Sánchez y J. Camacho-Tamayo. 2008. Variabilidad espacial de atributos físicos de un Typic haplustox de los llanos orientales de Colombia. Eng. Agríc. 28(1), 55-63.

Lal, R. y M.K. Shukla. 2004. Principles of soil physics. Marcel Dekker, New York, NY.

Safar, S., H. González y N. Cappelli. 2011. Efecto de los arados rotativos sobre algunas propiedades físicas de un suelo franco arcilloso. CES Medicina Veterinaria y Zootecnia 6(1), 32-44.

Siczek, A. y J. Lipiec. 2011. Soybean nodulation and nitrogen fixation in response to soil compaction and surface straw mulching. Soil Tillage Res. 114, 50-56.
Terminiello A., R. Balbuena, M. Ariata, J. Hilbert, J. Claverie J. y D. Jorajuria. 2007. Compactación inducida por el tránsito vehicular sobre un suelo en producción hortícola. Rev. Bras. Eng. Agríc. Amb. 4(2), 290-293. Doi: 10.1590/S1415-43662000000200027

Volveras, M. y C. Amezquita. 2009. Estabilidad Estructural del suelo bajo diferentes sistemas y tiempo de uso en laderas Andinas de Nariño, Colombia. Acta Agron. 58(1), 35-39.

Yu, W.Z., T. Liu, J. Gregory, G.B. Li, H.J. Liu, y J.H. Qu. 2012. Aggregation of nano-sized alum-humic primary particles. Sep. Purif. Technol. 99, 44-49. Doi: 10.1016/j.seppur.2012.08.017

Zhang, W., J. Crittenden, K. Li y Y. Chen. 2012. Attachment efficiency of nano-particle aggregation in aqueous dispersions: modeling and experimental validation. Environ. Sci. Technol. 46, 7054-7062. Doi: 10.1021/es203623z 